\title{
Construire la mémoire et l'identité autochtones aux États-Unis. Le documentaire photographique Project 562 de Matika Wilbur
}

Building Indigenous Memory and Identity in the USA. The photographic documentary Project 562 by Matika Wilbur

Construir la memoria y la identidad indígenas en los Estados Unidos. El documental fotográfico Project 562 de Matika Wilbur

\section{Aurélie Journée}

\section{OpenEdition}

\section{Journals}

Édition électronique

URL : http://journals.openedition.org/ideas/5342

DOI : $10.4000 /$ ideas.5342

ISSN : 1950-5701

\section{Éditeur}

Institut des Amériques

\section{Référence électronique}

Aurélie Journée, « Construire la mémoire et l'identité autochtones aux États-Unis. Le documentaire photographique Project 562 de Matika Wilbur », IdeAs [En ligne], 13 | 2019, mis en ligne le 01 mars 2019, consulté le 19 avril 2019. URL : http://journals.openedition.org/ideas/5342 ; DOI : 10.4000/ ideas. 5342

Ce document a été généré automatiquement le 19 avril 2019

\section{c)}

IdeAs - Idées d'Amériques est mis à disposition selon les termes de la licence Creative Commons Attribution - Pas d'Utilisation Commerciale - Pas de Modification 4.0 International. 


\title{
Construire la mémoire et l'identité autochtones aux États-Unis. Le documentaire photographique Project 562 de Matika Wilbur
}

\author{
Building Indigenous Memory and Identity in the USA. The photographic \\ documentary Project 562 by Matika Wilbur \\ Construir la memoria y la identidad indígenas en los Estados Unidos. El \\ documental fotográfico Project 562 de Matika Wilbur
}

\section{Aurélie Journée}

\section{Introduction}

1 Née en 1984, l'artiste Matika Wilbur (d'origine swinomish et tulalip1) grandit dans la réserve Swinomish, dans l'État de Washington. Sa passion pour la photographie naît lorsqu'elle est adolescente. A l'âge de dix-sept ans, la jeune femme intègre la Rocky Mountain School of Photography (dans le Montana), avant d'intégrer le Brooks Institute of Photography (en Californie), dont elle obtient une licence trois ans plus tard ${ }^{2}$.

Dix-sept ans, c'est aussi l'âge auquel l'artiste commence à suivre le séminaire de Craig Tanner, photographe de paysages. S'interrogeant sur les notions de «talent» et de "métier », son enseignement marque de façon durable l'esprit de Matika Wilbur.

Le corpus iconographique sur lequel porte cette étude rassemble des œuvres extraites de la série photographique Project $562^{3}$, qui a fait l'objet de l'exposition Seeds of Culture: The Portraits and Voices of Native American Women à l'Université de Harvard en 2016. À travers son analyse, notre étude a pour objectif principal d'interroger la notion de «documentaire photographique » à l'aune des images produites par une artiste nordamérindienne. Nous postulons que les caractéristiques formelles de ces récits visuels soulèvent, dans leurs contextes historique, social et culturel de production spécifique, des 
questionnements sur la construction de la mémoire et de l'identité des peuples autochtones aux États-Unis.

4 Notre analyse s'inscrit dans le cadre de recherches doctorales interrogeant le potentiel de la photographie à être un outil de revendications politiques et sociales dans une perspective décoloniale. Plus spécifiquement, nos travaux tendent à mettre en évidence que les artistes femmes nord-amérindiennes utilisent le médium photographique afin de livrer une critique de l'épistémologie des sciences et des techniques occidentales ayant nourri et légitimé la création et l'étude de la figure de l'« Indien » comme « Autre » (De l'Estoile B., 2010 [2007] ; Barthe C., 2006).

5 À partir d'une approche anthropologique, historico-culturelle et artistique de l'image photographique, nous étudierons d'abord le contexte de production de ce projet et ses objectifs. Ensuite, nous verrons en quoi son processus de création et ses modalités formelles réactualisent une pratique historiquement allochtone ${ }^{4}$ tout en mettant en valeur des cultures autochtones. En conclusion, nous ouvrirons notre réflexion sur ce projet en nous interrogeant sur ce qu'il révèle de la place et du rôle des femmes autochtones dans la société états-unienne contemporaine.

\section{Aux origines du projet Project 562}

6 Project 562 est le fruit d'une entreprise nomade. En 2014, Matika Wilbur se donne pour mission de parcourir le territoire des États-Unis, après avoir réuni quelques dollars suite à la vente de ses biens personnels. "[Son] voyage lui prendra plus de 3 ans. Durant les quinze premiers mois, elle a parcouru plus de 50000 miles, visité plus de 150 tribus [...] » (Newman Fricke S., 2014: 34). Elle a pour but de créer des enregistrements photographiques mais également sonores de ses contemporains autochtones, afin de déconstruire les stéréotypes auxquels ils sont confrontés, et promouvoir l'éducation. Pour ce faire, Matika Wilbur explore tout le champ des possibles grâce aux nouveaux médias, associant à l'utilisation d'un procédé photographique ancien - l'argentique -, la technologie numérique et les plateformes de financement participatif en ligne.

\section{A) Déconstruire les stéréotypes et promouvoir l'éducation}

7 Avec Project 562, Matika Wilbur tend à représenter grâce au médium photographique les 562 tribus amérindiennes que comptent les États-Unis ${ }^{5}$. L'artiste s'exprime ainsi : « Notre but est de dévoiler la véritable essence des questions amérindiennes, la beauté de la culture amérindienne, la grandeur de la tradition, et d'exposer sa vitalité ${ }^{\prime} »$. Comme l'explique Suzanne Newman Fricke: «Pour Wilbur, fournir des images de véritables autochtones contrebalance le nombre foisonnant de stéréotypes trouvés dans tous les médias, spécialement au cinéma, dans les photographies et dans les publicités » (Newman Fricke S., 2014).

8 L'artiste veut donner à voir ceux que l'on ne voit pas et/ou que la société refuse de rendre visibles. Dans son intervention donnée à Seattle en 2013, elle relève ainsi que :

Entre 1990 et 2000, 5868 superproductions hollywoodiennes ont été réalisées. Douze ont inclus des autochtones, toutes ont montré les autochtones comme étant spirituels et en contact avec la nature. Dix d'entre elles les ont montrés pauvres et abattus par la société. Dix les ont montrés comme se sentant en insécurité avec les blancs. (Wilbur M., 2013) 
Pour l'artiste, les membres des nations autochtones doivent être regardés différemment, et pour cela ne plus être représentés uniquement par des Occidentaux mais aussi et avant tout par des personnes autochtones dans un souci de souveraineté visuelle (Lidchi et al., 2009 ; Raheja, 2010). Pour déconstruire les fausses représentations, il faut donc parvenir à se représenter sans intermédiaires. En définitive, la question centrale irriguant la pensée de l'auteure de Project 562 est la suivante : "Comment pouvons-nous être vus comme un peuple moderne alors que nous sommes constamment vus comme les membres d'une race en voie de disparition revêtus de peaux tannées et de plumes?" (Wilbur M. in Newman Fricke S., 2014 : 34). L'expression « race en voie de disparition » est importante : elle se réfère à l'une des théories ayant servi à légitimer la colonisation au cours du XIX siècle, celle «de l'Indien en voie de disparition » ou "Vanishing Indian» (Parkman F., 1909 [1851] : 48), annonçant la disparition inéluctable des «tribus » amérindiennes ${ }^{7}$. Les valeurs d'authenticité et d'objectivité communément attribuées au médium photographique conduisent au développement de son utilisation systématique pour représenter cette « race en voie de disparition » ou « Vanishing Race» (Lyman C., 1982), immortalisée notamment par Edward Curtis. À rebours de cette pratique qui avait pour base idéologique la fin des peuples autochtones, Matika Wilbur crée quant à elle des images qui illustrent ce que le chercheur ojibwe Gerald Vizenor appelle la "survivance » amérindienne ${ }^{8}$ (Vizenor G. R., 1993, 1994). Ainsi, les images de cette artiste apparaissent comme de parfaits contre-exemples à l'idéologie de «l'Indien en voie de disparition » sur laquelle la domination coloniale a été fondée.

Si Project 562 vise à la déconstruction des stéréotypes, ce n'est pas son seul but. En effet, à travers cette série photographique, Matika Wilbur se donne également pour objectif d'éduquer à la fois les spectateurs et les sujets eux-mêmes, quant à la diversité et à la vitalité des cultures amérindiennes dans la société états-unienne contemporaine. Le public auquel se destine ce « documentaire photographique », dont «[...] le but ultime est l'éducation ${ }^{9}$, est pluriel et concerne à la fois les personnes de race blanche et les personnes autochtones.

11 Project 562 n'est pas la première série photographique de l'artiste consacrée à ce sujet. En effet, en 2004, Wilbur travaille sur Nous sommes un peuple, un projet visant à rendre hommage aux anciens des peuples de la côte Salish. Elle réalise ainsi leurs portraits, en se concentrant particulièrement sur les aînés Swinomish, Tulalip, Upper Skagit et Lummi. Dans Nous émergeons, elle porte un regard sur la façon dont les Amérindiens, pris dans une dualité culturelle, vivent dans la société états-unienne contemporaine. Enfin, son autre série Sauver l'Indien et tuer l'homme ${ }^{10}$, est un essai photographique qui répond aux tentatives de génocide et une traduction en images des voies que la guérison et la résilience autochtones ont empruntées au cours des dernières années (Newman Fricke S., 2014 : 34).

\section{B) Concilier photographie argentique et nouvelles technologies}

12 La richesse de Project 562 ne se situe pas seulement dans ses objectifs. En effet, des projets d'autres artistes, tels que Concrete Indians (2010) de Nadya Kwandibens (Ojibwe) et de sa société Red Works Photography, ou encore Native Americans: Keeping the Traditions Alive (2014) de Zoe Marieh Urness (d'origine tlingit et cherokee), répondent à peu près aux mêmes enjeux. 
13 En réalité, étudier l'œuvre de Matika Wilbur dans toute sa profondeur implique de se pencher davantage sur la technique spécifique de l'artiste. En effet, par l'adéquation de la technique utilisée aux questionnements que cette œuvre porte, Project 562 est un cas exemplaire de la mise de la forme au service du fond. La forme traduit l'association de l'enseignement classique reçu par l'artiste à des réflexions sur la mémoire et l'identité liées à sa culture, des premières étapes de sa réalisation aux rapports qui l'unissent à ses sujets.

14 Au cœur de la pratique de Matika Wilbur se situe la photographie argentique. Ce procédé est depuis la fin des années 1990 largement supplanté par la technologie numérique. Si le premier appareil photographique de l'artiste a été un Canon-EOS qui utilise une pellicule $35 \mathrm{~mm}$, elle a renoué avec un appareil très ancien pour Project 562, un Mamiya. Cette information technique est importante dans la mesure où ce type d'appareil a été utilisé par de grands noms de la photographie de paysages américains du $x x^{e}$ siècle, tels qu'Ansel Adams. Le Mamiya est en effet un «appareil photographique [qui] offre un champ de profondeur plus élevé et des textures plus riches » (Newman Fricke S., 2014: 35). De plus, l'artiste associe à l'utilisation de cet appareil et de plan-films la méthode dite du « Zone System ", technique développée par Ansel Adams lui-même, pour conférer à ces photographies ce rendu si particulier et créer une gamme de teintes plus dynamique dans les images.

Pour Project 562, Matika Wilbur personnalise cette technique réservée à la photographie argentique noir et blanc, en y ajoutant un procédé de colorisation manuel destiné à souligner certains éléments présents dans l'image (notamment certaines parties du corps ou des accessoires vestimentaires). "Elle prend souvent des photos avec du film noir et blanc, imprime les images, et ensuite les colorise partiellement avec de la peinture à l'huile. Si elle utilise du film couleur, elle manipule fréquemment les photographies sur Photoshop afin de dé-saturer l'arrière-plan ou renforcer la couleur à l'avant de l'image " (Walsh C., 2016). Grâce à une manipulation de l'image, elle procède ainsi à une interrogation du concept d'authenticité, qui était mis en avant par les photographes des $\mathrm{XIX}^{\mathrm{e}}$ et $\mathrm{Xx}^{\mathrm{e}}$ siècles tel que Curtis.

16 Enfin, l'autre particularité de Projet 562 est la manière avec laquelle le budget nécessaire à sa réalisation a été réuni. Matika Wilbur sollicite les formes les plus récentes de collecte de fonds, les plateformes de financement participatif en ligne. Elle opte ainsi pour le site Kickstarter, sur lequel elle lance sa première campagne en 2012, avant d'en créer une seconde en 2014. Le succès est inattendu, inespéré :

Le soutien pour son projet a été phénoménal. Elle réussit à lever des fonds pour son voyage grâce à deux campagnes sur Kickstarter. La première devait lever un montant initial de 30000 dollars pour que Wilbur puisse commencer à voyager ; le 1 er novembre 2012, la première campagne fut lancée et récolta 35428 dollars. La seconde campagne avait un objectif plus ambitieux de 54000 dollars; le 20 février 2014, la campagne a atteint son but, récoltant la somme incroyable de 213461 dollars. (Newman Fricke S., 2014 : 35)

17 Le projet de Matika Wilbur bénéficie également d'une couverture médiatique importante, sur le site web Upworthy ou encore à la télévision, sur NBC News. À travers son titre, qui traduit une volonté d'exhaustivité, et le voyage qu'il implique, Project 562 rappelle le travail monumental d'Edward S. Curtis, The North American Indian ${ }^{11}$. Matika Wilbur le connait mais refuse de voir son œuvre y être comparée :

Au cours des quinze dernières années, j'ai photographié et partagé des histoires provenant des communautés autochtones des États-Unis. Depuis 2012, Project 562 
m'a permis de faire ce travail avec des personnes de tout le pays et j'ai été dans près de 400 tribus. Dans les prochaines, j'espère avoir accompli ma mission. À cause de ma dévotion à photographier des autochtones, des gens m'ont surnommée «La Curtis des Temps Modernes ». À chaque fois que j'entends cela, ça me donne envie de vomir. (Wilbur M., 2018)

De manière très directe, Matika Wilbur exprime le dégoût que la comparaison avec Edward Curtis lui inspire. Non pas tant en raison d'un rejet esthétique mais de l'idéologie sous-jacente aux photographies réalisées à l'époque du photographe.

\section{C) Déconstruire les représentations occidentales du passé ?}

19 Pourquoi donc vouloir rompre si radicalement avec une œuvre si conséquente? Les images sépia d'Edward Curtis ont nourri un pictorialisme photographique à l'esthétique romantique intemporelle (Arrivé M., 2012 ; Gidley M., 1994).

Curtis a été financé par J.P. Morgan pour photographier «la race en voie de disparition ». Ce mercenaire photographique a été le premier à détourner l'image de l'Indien, célèbre à travers tout le Pays Indien pour représenter de façon artificielle ses sujets avec des objets et des vêtements qui appartenaient à différentes régions tribales. Il a dû donner des instructions à ses sujets pour qu'ils se tiennent loin des infrastructures modernes, dans le but de capturer leurs qualités de «sauvages » et leurs modes de vie (Wilbur M., 2018).

Ce faisant, cette œuvre a contribué sans aucune commune mesure à créer, mythifier et rigidifier la figure de "l'Indien». Si l'objectif initial de Curtis est de rassembler les preuves de l'existence passée de peuples qui ne seraient plus, et de faire l'inventaire de pratiques culturelles et vestimentaires considérées à l'époque comme exotiques, il n'en reste pas moins que ce photographe a sorti une partie des peuples autochtones d'Amérique de la frise du temps. Il les a soustraits à l'Histoire, les a gelés dans un présent éternel en les écartant du contemporain ou plutôt de l'actuel. Au début du $\mathrm{Xx}^{\mathrm{e}}$ siècle, cette esthétique s'accompagne de nouveaux récits soulignant la peur présumée des Amérindiens à l'égard du médium photographique, qu'ils considèreraient comme un « voleur d'ombre », ou « shadow-catcher » (Jackson, W.H, 1940).

Ici, ce n'est pas tant la véracité de cette croyance qui nous intéresse que l'écho qu'elle a trouvé dans la construction des photographies de l'époque. La pratique du médium cristallise alors les rapports de domination existant entre colonisateurs et colonisés. Les sujets photographiés étaient placés dans une situation de subordination par rapport au photographe blanc, qui déterminait à la fois les positions, les attitudes et les attributs vestimentaires dont se paraient les sujets. À la véracité ou l'authenticité du discours émanant de la représentation se substituent donc les désirs esthétiques du photographe, dont l'objectif n'est autre que de faire de « belles images » répondant au goût du moment. Ce dernier cultive ainsi la fascination pour l'exotique des collectionneurs et amateurs européens, notamment pour les cartes postales qui sont faciles à diffuser.

L'on peut ainsi s'interroger sur la représentation de l'individualité des sujets dans ces images, qui nourrissaient davantage une uniformisation de l'identité amérindienne plutôt que la mise en évidence de sa pluralité. Comme l'indique l'auteure de Project 562 :

Il [Curtis] intitula ces images par exemple "Indien \#3», "Femme Chumash», et "Chasseur de têtes", comme si les personnes sur ces photos n'avaient pas de noms, comme s'ils ne méritaient pas d'être différenciés les uns des autres. (Wilbur M., 2018) 
La pratique photographique d'Edward S. Curtis, et l'esthétique qui la caractérise, n'auraient-elles finalement pas « volé l'âme » des membres des peuples photographiés, en ne rendant pas compte du caractère unique de chacun? C'est précisément ce avec quoi Matika Wilbur cherche à rompre, lorsqu'elle déclare : «Je veux [...] des portraits d'étude d'un personnage authentique qui essayent vraiment de capturer l'essence et l'âme d'une personne » (Corrie J., 2015). Or, les touches de couleur distillées par l'artiste sur ses photographies tendent à mettre en exergue cette idée d' "authenticité » conjuguée au présent, ou plus exactement de «survivance» telle que mentionnée par Gerald R. Vizenor. Ainsi, les images de Matika Wilbur tendent à rompre avec les premières représentations d'autochtones connues et réalisées par des photographes occidentaux - bien avant Curtis -, historiquement en noir et blanc, sur un fond neutre (drap blanc) ou un décor restituant une nature artificielle. Matika Wilbur substitue à ces ersatz de paysages des vues réalisées "sur le motif », en prise directe avec le réel :

J'ai décidé [...] de photographier les gens dehors dans leur environnement naturel parce que je pense que [...], cela fait aussi sens de montrer un paysage car le paysage est aussi connecté à la perception que nous avons de nous-mêmes. [D'où ils viennent] est aussi important pour raconter l'histoire (Corrie J., 2015).

Avec Project 562, Matika Wilbur réalise donc une série photographique dont les images sont à mi-chemin entre le portrait et la photographie de paysages. Nous allons maintenant voir en quoi la combinaison des deux sert la construction de la mémoire et de l'identité des autochtones aux États-Unis.

\section{Entre portraits et photographies de paysages : un projet à l'" esthétique du recensement » entre rupture et continuité}

Le terme de «recensement» est une notion sociologique / démographique n'ayant a priori rien à voir avec l'art. Pourtant, cette activité entretient des liens historiques avec la photographie, par le biais notamment des études portant sur les différents acteurs sociaux et leurs territoires, dont les autochtones, lesquelles ont conduit à la systématisation de la réalisation de portraits. Leur esthétique varie en fonction de leurs auteurs et de leurs contextes de production. Nous allons voir comment Matika Wilbur, à travers Project 562, s'inscrit en rupture et en continuité avec ces études.

\section{A) Rompre avec la photographie romantique pictorialiste d'Edward Curtis}

À travers Project 562, Matika Wilbur veut s'affranchir de deux genres photographiques : les portraits ethnographiques d'Amérindiens réalisés pendant les grandes explorations et les missions scientifiques, tels que les « types coloniaux » et les portraits de Curtis, construits selon l'idéologie du "Vanishing Indian», et les photographies de paysages d'Ansel Adams. En effet, si le travail de ce dernier a influencé l'œuvre de Matika Wilbur, nous allons voir qu'il a en réalité nourri l'idée de la "découverte» de la terra incognita, en répondant à la quête d'une certaine « naturalité ». 

des souhaits et des préférences des personnes qu'elle photographie, Matika Wilbur fait du documentaire photographique une entreprise qui ne fonctionne pas dans un sens unique : la photographe n'impose pas ses choix à celles et ceux qu'elle photographie mais les inclut à chaque étape du processus conduisant à l'objet final. Ainsi, afin de déconstruire une forme de cannibalisme photographique occidental, qui consomme les « Autres » plus qu'il ne les représente dans leur réalité, l'artiste développe une démarche plaçant en son cœur la volonté du sujet. C'est ce qu'elle appelle elle-même "la méthode de la photographie autochtone» ("the indigenous photography method») précisant que cela l'oblige à devenir une meilleure photographe. «Maintenant je réalise le cliché quand les gens sont prêts, non quand je le ressens, ou quand le soleil est bon ", dit Matika Wilbur. "C'est un tournant dans la façon dont je perçois ce que je fais » (Walsh C.). Au lieu de s'interroger sur la façon d'obtenir le meilleur cliché, elle demande à ses sujets le lieu dans lequel ils voudraient que leur photographie soit prise, ce qu'ils souhaitent porter, ou encore l'histoire qu'ils aimeraient raconter par l'entremise de son objectif. Elle ajoute, à propos de la photographie en studio : «C'est l'intimité du studio, la présence de l'appareil photographique et la connexion avec ce que le sujet ressent avec moi qui permet que l'honnêteté et la vulnérabilité soient présentes dans chaque image » (Newman Fricke S., 2014 : 34).

Si Matika Wilbur se distingue de ses prédécesseurs par sa façon de réaliser ses portraits, elle s'en différencie également à travers la façon dont elle décide de faire de ses sujets des habitants.

\section{B) Ancrer l'être humain dans son environnement : matérialiser les liens des peuples autochtones avec la nature}

31 La composition des photographies de la série Project 562 est particulière dans la mesure où le paysage occupe toujours près de la moitié de l'image (l'autre moitié étant réservée au portrait). Selon nous, cela traduit l'influence exercée par Ansel Adams sur Matika Wilbur.

Néanmoins, aux $\mathrm{XIX}^{\mathrm{e}}$ et $\mathrm{XX}^{\mathrm{e}}$ siècles, les photographies de paysages sont presque systématiquement dépourvues d'êtres humains. Au sens propre, ces photographies ne sont pas incarnées. Les images d'Ansel Adams, par exemple, donnent à voir l'immensité des espaces naturels, des Grands Parcs, aux États-Unis, en la coupant de toute dimension humaine et/ou animale. Dans ces photographies, la nature est représentée comme majestueuse, mais précisément parce qu'elle n'est pas habitée. Elle est brute. John Szarkowski dira même de ces images: "[Dans l'Ouest] le paysage était si dénué de références humaines, si dépourvu de signes d'histoire, de philosophie et de poésie, que les valeurs plastiques étaient les seules disponibles ${ }^{12}$.» 
images se prêtent ainsi à une lecture complexe, car, tout en mettant en avant la beauté de la nature laissée à l'état sauvage, elles laissent entrevoir aux spectateurs de l'époque l'idée de terres qui resteraient à conquérir. Tout serait encore à y faire, puisque ces espaces ne seraient pas encore domptés, maîtrisés, « acculturés ». En d'autres termes, les images par lesquelles sont connues la grandeur et la magnificence des grands parcs nationaux états-uniens, aussi belles soient elles, ne sont jamais que les reflets du mythe de la terra incognita sur laquelle ont été bâties la colonisation et les tentatives de génocide. place fondamentale aux paysages, puis en intervenant directement sur ses images à travers le procédé de colorisation manuelle, Matika Wilbur réincarne cette nature qui a été présentée comme "vierge » mais est en réalité meurtrie et violée - à l'instar des femmes autochtones ${ }^{13}$. Elle donne à voir les personnes qui les premières l'ont habitée, et intensifie même l'idée de " présence » amérindienne (Vizenor G., 1998). En effet, grâce à l'intervention directe sur les images que constitue la colorisation manuelle, l'artiste interroge finalement comment la colonisation et les théories sur lesquelles elle repose se sont accompagnées de la production d'images témoins de la volonté d'intervenir tant sur les corps que sur les territoires. L'action coloniale s'est déroulée de manière concomitante sur les êtres et sur les terres, la colonisation des uns entraînant nécessairement la colonisation des autres.

À travers Project 562, ce sont donc les images produites dans le contexte idéologique du «Vanishing Indian» et de la «terra incognita» que Matika Wilbur apparaît vouloir déconstruire. Mais l'artiste a également été inspirée par d'autres photographes de paysages, tels que Dorothea Lange (Newman Fricke S., 2014: 39). Cette référence est intéressante, en ce que, précisément, celle-ci n'a pas été seulement une photographe de paysages mais bien une grande portraitiste, symbole de la photographie de "style documentaire » des années 1930 aux États-Unis (Lugon O., 2002).

\section{C) Project 562, un projet de « style documentaire »?}

En marge des photographies romantiques de Curtis et de celles de paysages d'Anse Adams, les années 1930 aux États-Unis sont marquées par le développement d'une "photographie sociale», dont les séries de la Farm Security Administration (FSA) témoignent. Celles-ci mettent à contribution de célèbres photographes, tels que Walker Evans et Dorothea Lange ${ }^{14}$. Les images alors réalisées documentent les effets de la crise économique de 1929 et de la Grande Dépression sur la population et accompagnent la campagne éducative de la FSA. Ainsi, ces photographies se caractérisent par une sorte d'esthétique de la trace qu'implique l'exode grandissant des ruraux, qui privilégient les villes au détriment des campagnes. Les séries photographiques alors réalisées traduisent une obsession du temps qui passe, de la disparition, de la désuétude, avec cette idée majeure de constituer des images pour les générations futures.

À travers Project 562, Matika Wilbur montre que sa conception du «documentaire photographique » se rattache aux pratiques de "style documentaire " pour trois raisons. D'une part, cette œuvre illustre une volonté de constituer des traces visuelles (à l'instar d'Evans ou de Lange), de rendre compte, de témoigner, d'écrire l'histoire d'un groupe, ici celui des peuples amérindiens dans la société états-unienne contemporaine. D’autre part, un parallèle peut être fait entre le caractère nomade de la démarche de Wilbur et l'esthétique de la trace des photographies d'exode de la FSA. En effet, chacune de ces 
pratiques est caractérisée par un mouvement, par un déplacement, qui rappelle l'histoire amérindienne et les déplacements de peuples autochtones qui la constituent. Nous pensons notamment à l'Indian Removal Act (1830) à l'origine du Trail of Tears (Piste des Larmes) ${ }^{15}$.

Enfin, si Project 562 répond à des objectifs sociaux, reposant à la fois sur une démarche mémorielle (constituer les traces de peuples) et identitaire (montrer ce que sont les Amérindiens contemporains à travers leurs pratiques vestimentaires et leurs relations à la terre), cette série affirme également à travers son processus de création et sa technique (le procédé de colorisation manuelle, entre autres) que la composante artistique est fondamentale.

Or, si l'on se remémore la vie des images d'Evans par exemple, Project 562 apparaît suivre le parcours inverse. Alors que les photographies produites pour la FSA étaient réalisées dans une perspective documentaire puis exposées par certains commissaires d'expositions tels que John Szarkowsk ${ }^{16}$ pour leurs qualités artistiques, les images de Project 562 sont pensées dès le début par l'artiste en tant qu'œuvres, mobilisant des techniques artistiques tel que le procédé de colorisation manuelle à la peinture à l'huile. Ainsi, Matika Wilbur utilise les valeurs associées au «style documentaire » et accentue l'ambiguïté des relations photographie-art qui le caractérise. Elle se réapproprie l'histoire de la photographie et de l'art, longtemps exclusivement écrites par des Occidentaux.

Matika Wilbur semble aussi vouloir, grâce au Project 562, substituer au caractère statique des photographies de Curtis des questions liées à la perception de soi, de la culture et des traditions. L'artiste a à cœur de représenter des hommes et des femmes amérindien.ne.s - et surtout des femmes - en conjuguant leur existence au présent.

41 À travers cette œuvre se pose aussi la question de la décolonisation des représentations, et notamment des femmes amérindiennes. Pour ce faire, Matika Wilbur replace au cœur de ses images les spiritualités autochtones. Les femmes amérindiennes photographiées ne sont pas réduites à de simples spécimens comme elles ont pu l'être dans les photographies du XIX ${ }^{e}$ siècle dont les légendes, bien souvent, ne les désignaient que sous l'appellation « X's squaw » (ou « squaw de X ») ${ }^{17}$, sans aucune individualisation et surtout révélant la pensée patriarcale. Dans Project 562, chacune des photographies est légendée avec le nom et le prénom de la femme photographiée. D'autre part, la femme dans ce projet n'est pas réduite à un stéréotype hypersexualisé répondant au schème de pensée hétéronormatif, contrairement à ce qui a été diffusé par la littérature et le cinéma depuis le $\mathrm{XIX}^{\mathrm{e}}$ siècle $^{18}$. Enfin, à travers une démarche réflexive dont la pratique centrale place les traditions et les liens unissant les femmes autochtones à leurs terres, à la Terre, Matika Wilbur crée des images qui illustrent une forme de féminisme nord-amérindien plus proche du motherism africain (Acholonu C., 1995) ${ }^{19}$ que du féminisme hégémonique blanc, insistant sur les relations de la femme à la terre comme mère (« Mother Earth » ou TerreMère) mais également sur la complémentarité des êtres humains plutôt que sur leurs différences en termes de sexe et/ou de genre.

\section{Conclusion}

42 À travers une série de portraits de femmes et d'hommes réalisés à l'aide des moyens technologiques les plus récents, tout en faisant référence à une culture visuelle et 
matérielle antérieure, Matika Wilbur s'affirme comme une artiste femme autochtone ayant à cœur de décoloniser les représentations des Amérindiens.

Pourtant, il est intéressant de constater que les témoignages de Matika Wilbur citent principalement comme références des photographes hommes, et très peu de femmes ${ }^{20}$. Gertrude Käsebier, par exemple, a consacré une grande partie de sa production aux photographies d'Amérindiens (Michaels B.L. et Käsebier G., 1992). Elle ne cite pas de photographes autochtones, qui pourtant ont commencé très tôt à développer une pratique photographique, tels que Richard Throssel (1882-1933), Cree adopté par la communauté Crow qui notamment documenté la réserve Crow au début du $\mathrm{xx}^{\mathrm{e}}$ siècle, Peter Pitseolak (Inuit, 1902-1973) et Horace Poolaw (Kiowa, 1906-1984). En effet, l'artiste ne mentionne aucun photographe amérindien qui aurait pu guider ses choix formels. Ceci révèle que les écoles de photographie, et plus généralement d'art, même actuelles, aux États-Unis, ne laissent qu'une très faible part à l'enseignement de l'histoire de l'art et de la photographie autochtones, et continuent à nourrir l'idée d'une histoire de l'art écrite par et pour des Occidentaux. Project 562 peut aussi être lu à l'aune du travail de la photographe cherokee Jennie Ross Cobb, qui documente la vie de sa nation dès la fin du $\mathrm{XIX}^{\mathrm{e}}$ siècle $^{21}$. D'autre part, si Matika Wilbur vise à donner un visage à la contemporanéité de la vie autochtone aux États-Unis, nous nous interrogeons sur la façon dont elle utilise, pour une déconstruction décoloniale, des observations presque essentialistes : « Notre but est de dévoiler la véritable essence des questions amérindiennes, la beauté de la culture amérindienne, la grandeur de la tradition, et d'exposer sa vitalité2 ${ }^{22}$.» L'artiste parle d'« essence ", et n'utilise que des articles au singulier ("la beauté », « la culture », «la tradition »), mettant de côté précisément la diversité que son projet tend à mettre en exergue en voulant photographier chacun des peuples autochtones aux États-Unis.

En conclusion, Project 562 montre que la construction de la mémoire et de l'identité autochtones aux États-Unis est complexe en ce qu'elle implique plusieurs strates, plusieurs niveaux de lecture. Les Amérindien.ne.s sont pris.e.s dans une tension constante, les écartelant entre la volonté de perpétuer leurs traditions et de vivre dans le présent, alors même que ces notions de «traditions » et de «présent » traduisent une vision linéaire du temps occidental qui n'est pas la vision cyclique du temps des peuples autochtones. La difficulté est aussi de se construire à partir de références occidentales - tant historiques et culturelles qu'artistiques-, les politiques d'assimilation étatsuniennes entre 1790 et 1920, puis jusqu'à la fin des années 1970 avec les écoles résidentielles, ayant travaillé activement à l'éradication de toute mémoire et identité culturelle autochtones. Project 562 combine toutes ces tensions, mais également toutes ces tentatives de vivre sa culture dans une société capitaliste et patriarcale blanche qui érige, au nom de l'universalisme, l'étendard de l'homogénéité. Grâce au médium photographique et aux enregistrements sonores qu'elles constituent dans le cadre de Project 562, Matika Wilbur offre finalement aux femmes autochtones une occasion unique d'ouvrir leur voix ${ }^{23}$. 


\section{BIBLIOGRAPHIE}

Acholonu, Catherine, Motherism: the Afrocentric Alternative to Feminism, Owerri, Afa Publications, 1995.

Agee, James (texte) et Evans, Walker (photographies), Louons Maintenant les Grands Hommes [Let Us Now Praise Famous Men], Paris, Plon, coll. « Terre Humaine », 1972 [1941].

Arrivé, Mathilde, « Par-delà le vrai et le faux ? Les authenticités factices d'Edward S. Curtis et leur réception ", Études photographiques [En ligne], n 29, 2012, mis en ligne le 05 février 2013. URL : http://etudesphotographiques.revues.org/3283. Page consultée le 16 novembre 2017.

Barthe, Christine, D'un regard l'Autre, photographies du XIX siècle, ouvrage publié à l'occasion de l'exposition organisée au musée du quai Branly à Paris du 18 septembre 2006 au 21 janvier 2007, Paris, coédition Musée du Quai Branly - Actes Sud, 2006.

Brunet, François, « La photographie de l'ouest ou l'histoire expérimentale du paysage », Revue française d'études américaines, n² 26, Le paysage américain, novembre 1985, p. 417-429.

Churchill, Ward, Kill the Indian, Save the Man: The Genocidal Impact of American Indian Residential Schools, San Francisco, City Lights Books, 2004.

Corrie, Jennifer, « Matika's story. The woman behind the FSU Fine Art exhibit » [en ligne], Torch Ferry State University website. Source URL : http://fsutorch.com/2015/09/15/matikas-story/. Page consultée le 26 décembre 2016.

Curtis, Edward S., The North American Indian, 20 volumes, 2200 photographies, 1907-1930.

De l'Estoile, Benoît, Le Goût des Autres. De l'Exposition coloniale aux Arts premiers, Paris, Flammarion, coll. « Champs Essais », 2010 [2007].

Gidley, Mick, « Pictorialist Elements in Edward S. Curtis's Photographic Representation of American Indians ", The Yearbook of English Studies, vol. 24, Ethnicity and Representation in American Literature, 1994, p. 180-192.

hooks, bell, De la marge au centre. Théorie féministe, Paris, Cambourakis, coll. « Sorcières », 2017 [1984]. Traduit de l'anglais par Noomi B. Grüsig.

Jackson, William Henry, Time Exposure: The Autobiography of William Henry Jackson, New York, G. P. Putnam's sons, 1940.

Lidchi, Henrietta, Tsinhnahjinnie, Hulleah J., Visual Currencies : Reflections on Native Photography, Edinburgh, National Museums Scotland, 2009.

Lugon, Olivier, Le Style documentaire. D'August Sander à Walker Evans: 1920-1945, Paris, Macula, 2002.

Lyman, Christopher M., The Vanishing Race and Other Illusions: Photographs of Indians by Edward S. Curtis, New York, Pantheon (in association with the Smithsonian Institution Press), 1982.

Michaels, Barbara L. et Käsebier, Gertrude, Gertrude Käsebier: The Photographer and her photographs, New York, Harry N. Abrams, 1992.

Newman Fricke, Suzanne, « Swinomish-Tulalip Photographer Matika Wilbur Profile », First American Art Magazine, $\mathrm{n}^{\circ}$ 3, été 2014. 
Parkman, Francis, The Conspiracy of Pontiac and the Indian War after the Conquest of Canada, t. I, « To the Massacre at Michillimackinac », Boston, Little Brown, 1909 [1851].

Raheja, Michelle H., Reservation Reelism : Redfacing, Visual Sovereignty, and Representations of Native Americans in Film, Lincoln, University of Nebraska Press, 2010.

Vizenor Gerald R., Manifest Manners: Post indian Warriors of Survivance, Hanover, Wesleyan University Press, 1994.

Vizenor Gerald R., « The Ruins of Representation: Shadow Survivance of the Literature of Dominance », American Indian Quaterly, vol. 17, n 1, hiver 1993, p. 7-30.

Vizenor Gerald R., Fugitive Poses. Native American Indian Scenes of Absence and Presence, Lincoln (NE), University of Nebraska Press, 1998.

Walsh, Colleen, «Photographing Native American culture », The Harvard Gazette [en ligne]. Source URL : http://news.harvard.edu/gazette/story/2016/05/photographer-adds-clarity-insight-tocontemporary-native-american/. Page consultée le 14 juin 2018.

Wilbur, Matika, «Edward S. Curtis. Again?», blog Project 562, mis en ligne le 8 mai 2018. Source URL : http://www.project562.com/blog/edward-s-curtis-again/. Page consultée le 18 février 2019.

Woods, Daniel R., Reclaiming the Voice : Gerald Vizenor. Literary Trickster in a Postmodern Language Game, Radford, Radford University, 2003.

$\mathrm{Yu}$, Jane, « Kill the Indian, Save the man » [article en ligne], publié au printemps 2009. Source URL : http://pabook2.libraries.psu.edu/palitmap/CarlisleIndianSchool.html. Page consultée le 07 février 2017.

\section{NOTES}

1. Les nations amérindiennes Swinomish et Tulalip se situent dans la région du Puget Sound, dans le Nord-Ouest des États-Unis (État de Washington) et au Sud-Ouest du Canada (ColombieBritannique).

2. Site internet de l'artiste Matika Wilbur : http://www.matikawilbur.com/. Page consultée le 18 octobre 2016.

3. Site internet de Project 562 : http://www.project562.com/. Page consultée le 14 octobre 2016.

4. Voir par exemple: Edward S. Curtis, The North American Indian, 20 volumes, 2200 photographies, 1907-1930.

5. Le nombre de tribus officiellement reconnues par le gouvernement fédéral états-unien s'élève aujourd'hui à 567 . Il ne reflète néanmoins pas l'entière diversité des peuples autochtones dans le pays, qui seraient plus de 2500 .

6. Site internet de l'artiste Matika Wilbur : http://www.matikawilbur.com/. Page consultée le 14 octobre 2017. Traduit de l'anglais par l'auteure.

7. L'historien Francis Parkman écrit en 1851 que « l'aborigène était par nature inchangeable et destinée à l'extinction». Traduction de l'anglais par l'auteure («the aborigine was by nature unchangeable and by fate doomed to extinction.»). Source: Yu, Jane (2009). Nous utiliserons désormais plus volontiers la notion de «peuples» que celle de «tribus», suivant en cela la dénomination de l'ONU, depuis notamment l'adoption de la Déclaration des Droits des Peuples Autochtones en 2007.

8. «La survivance est une forme active de la présence, la pérennité d'histoires traditionnelles, pas une simple réaction ou un nom survivant. Les histoires de survivance autochtone sont des 
renonciations à la domination, à la tragédie et à la victimisation. La survivance signifie le droit de succession ou le retour à un état et en ce sens, l'état de survivance autochtone »: Gerald R. Vizenor cité par Daniel R. Woods (2003:6). Traduction de l'anglais au français par l'auteure.

9. Site internet de l'artiste Matika Wilbur : http://www.matikawilbur.com/.

10. Ce titre détourne la célèbre phrase de Richard Henry Pratt « Kill the Indian and Save the Man " (« Tuer l'Indien et Sauver l'Homme»). À ce sujet, voir notamment: Ward Churchill (2004), quoique cet auteur soit controversé quant à la question du génocide indien.

11. Euvre réalisée entre 1907 et 1930, composée de 20 volumes et réunissant plus de 2200 photogravures.

12. John Szarkowski, American Landscapes (exposition du Museum of Modem Art), New York, 1981, p. 5 et 7, cité par François Brunet (1985: 419).

13. Matika Wilbur l'exprime ainsi: «Une femme a raconté avoir été stérilisée sans son consentement. Une autre a dit avoir été violée alors qu'elle était dans l'Armée puis rejetée par sa propre communauté après qu'un enfant métis est né de cette agression », citée par Colleen Walsh (2016). Traduit de l'anglais par l'auteure.

14. Voir par exemple: Agee, James (texte), Evans, Walker (photographies) (1972 [1941]). L'œuvre s'ouvre sur cette phrase-manifeste: «Faisons l'éloge de ces personnages glorieux qui sont nos ancêtres ", qui n'est pas sans faire écho à la démarche de Matika Wilbur que cristallise Project 562. 15. Série de relocalisations forcées ayant eu lieu entre 1831 et 1838 , à la suite de l'adoption de l'Indian Removal Act.

16. Comme le montre la rétrospective du travail de Walker Evans qu'il organise au MoMA en 1971.

17. Par « $\mathrm{X}$ » nous désignons le nom du mari de la femme autochtone représentée. Voir, par exemple, les albums photographiques de W. H. Jackson conservés au musée du quai BranlyJacques Chirac (étudiés par l'auteure durant un stage effectué au service iconographique entre octobre 2014 et février 2015).

18. À ce sujet, voir par exemple l'étude du cas particulier de la White Squaw, qui a fait l'objet d'une œuvre de l'artiste amérindienne crow Wendy Red Star et de notre communication « Tuer Pocahontas. Déconstructions et reconstructions identitaires dans l'œuvre de Wendy Red Star ", à l'occasion $\mathrm{du}$ colloque international Subjectivités féministes, queer et postcoloniales en art contemporain. Une histoire en mouvements, Université Rennes 2, Campus Villejean, 8-10 avril 2015.

19. «Le féminisme africain-américain, qui accepte la définition de Walker et le nouveau nom de baptême pour la lutte des femmes ne peuvent être un havre acceptable pour le féminisme africain ", citée par Nassira Hedjerassi dans la préface de l'ouvrage de bell hooks (2017 [1984] : 25).

20. Seule la femme photographe Joyce Tenneson est citée: «Parmi les photographes plus contemporains, j'adore Joyce Tenneson, son livre de portraits Wise Women » (Newman Fricke S., 2014 : 40). Traduit de l'anglais par l'auteure.

21. Une thèse sur cette photographe est en cours de préparation depuis 2013 : Emma Doubts, « A Humanizing Eye: Situating the snapshot photography of Jennie Ross Cobb, 1890-1910», Université de Sussex.

22. Site internet de Project 562 : http://www.project562.com/. Page consultée le 14 octobre 2016.

23. Ouvrir la Voix, film écrit et réalisé par Amandine Gay, Bras de Fer Production et Distribution, 2017, 122 min. 


\section{RÉSUMÉS}

À travers l'analyse de la série photographique Project 562 de l'artiste nord-amérindienne Matika Wilbur, notre étude a pour objectif principal d'interroger la notion de «documentaire photographique " à l'aune de représentations autochtones réflexives. Nous postulons que les caractéristiques formelles de ces récits photographiques soulèvent, dans leur contexte historicoculturel de production spécifique, des questionnements sur la construction de la mémoire et de l'identité des Amérindiens aux États-Unis. Notre analyse s'inscrit dans le cadre de recherches doctorales qui interrogent le potentiel de la photographie à être un outil de revendications politiques et sociales. Plus spécifiquement, nos travaux tendent à mettre en évidence que les artistes femmes autochtones en Amérique du Nord utilisent le médium photographique afin de livrer une critique de l'épistémologie, des sciences et des techniques occidentales, dans une perspective décoloniale. Cet article entend ainsi retracer les origines de l'œuvre Project 562 de Matika Wilbur (artiste d'origine tulalip et swinomish) afin d'en étudier les modalités particulières et comprendre ses objectifs. Il s'agira enfin de voir en quoi les images réalisées, à mi-chemin entre la photographie de portraits et celle de paysages participent d'une «esthétique du recensement» entre rupture et continuité, interrogeant la terminologie même de «photographie documentaire». À partir d'une approche historique, anthropologique et culturelle de l'image photographique, nous étudierons d'abord le contexte de production de ce projet et ses objectifs. Ensuite, nous verrons en quoi son processus de création et ses modalités formelles propres réactualisent une pratique historiquement allochtone tout en valorisant des cultures autochtones. En conclusion, nous nous interrogerons sur ce que ce projet révèle de la place et du rôle de la femme autochtone dans la société contemporaine.

Through the analysis of the photographic series Project 562 created by Indigenous North American artist Matika Wilbur, our study aims to question the concept of «photographic documentary » regarding Indigenous representations of themselves. Our presumption is that the formal aspects of these photographic narratives raise, in their specific historical and cultural contexts of production, issues about Indigenous memory and identity in the United States. Our analysis is linked to our $\mathrm{PhD}$ research that questions the potential of photography as a tool for political and social claims. More specifically, our research tries to highlight that Indigenous women artists in North America use the photographic medium in order to criticize Western epistemology, sciences and techniques from a decolonial perspective. This paper aims to trace, through an historical, anthropological and cultural approach of the photographic image, the context of production of Matika Wilbur's Project 562 and its goals. Then, we will see how its creative process and its own formal modalities update a historically Western practice while valuing Indigenous cultures. In conclusion, we will question what this project reveals about the place and role of Indigenous women in contemporary society.

El objetivo de nuestro artículo es interrogar la noción de "documental fotográfico" a la luz de las representaciones autóctonas reflexivas, gracias a la serie fotográfica Project 562 de la artista indígena norteamericana Matika Wilbur. Postulamos que las características formales de estos relatos fotográficos reflejan, en su contexto histórico y cultural de producción, la construcción de la memoria y de la identidad de los indígenas en los Estados Unidos. Este análisis hace parte de 
nuestra investigación doctoral que se concentra en el potencial de la fotografía como instrumento de reivindicaciones políticas y sociales. Este artículo trata de mostrar, en particular, que las artistas indígenas en América del Norte utilizan la fotografía para criticar, desde una perspectiva descolonizadora, la epistemología, las ciencias y las técnicas occidentales. Adoptando un enfoque histórico, antropológico y cultural de la imagen fotográfica, estudiaremos el contexto de producción y los objetivos del Project 562. Veremos, pues, cómo su proceso creativo y sus características formales actualizan una práctica históricamente occidental para valorizar, al contrario, culturas autóctonas. En conclusión, interrogaremos la manera en que este proyecto revela el protagonismo y el papel de la mujer indígena en la sociedad contemporánea.

\section{INDEX}

Palabras claves : fotografía, fotografía documental, indígenas, Estados Unidos, identidad Keywords : photography, documentary photography, indigenous peoples, United States, identity Mots-clés : photographie, photographie documentaire, autochtone, États-Unis, identité

\section{AUTEUR}

\section{AURÉLIE JOURNÉE}

Doctorante en Anthropologie sociale et Ethnologie, École des Hautes Etudes en Sciences Sociales, Laboratoire d'Anthropologie Sociale. Elle prépare une thèse intitulée « Artistes femmes et queer autochtones en Amérique du Nord face à leur(s) image(s), des années 1970 à nos jours. Une histoire intersectionnelle et décoloniale des arts contemporains autochtones aux États-Unis et au Canada ». aurelie.journee@hotmail.fr 